\title{
A New Image Denoising Method Based on Adaptive Multiscale Morphological Edge Detection
}

\author{
Gang Wang, ${ }^{1}$ Zesong Wang, ${ }^{2}$ and Jinhai Liu ${ }^{1}$ \\ ${ }^{1}$ School of Information Science and Engineering, Northeastern University, Shenyang, Liaoning 110819, China \\ ${ }^{2}$ Institute of Petroleum and Chemical Industry, Shenyang University of Technology, Shenyang, Liaoning 110870, China \\ Correspondence should be addressed to Gang Wang; gangwang@ise.neu.edu.cn
}

Received 18 August 2016; Revised 29 January 2017; Accepted 2 March 2017; Published 5 April 2017

Academic Editor: Lotfi Senhadji

Copyright (c) 2017 Gang Wang et al. This is an open access article distributed under the Creative Commons Attribution License, which permits unrestricted use, distribution, and reproduction in any medium, provided the original work is properly cited.

\begin{abstract}
Wavelet transform is an effective method for removal of noise from image. But traditional wavelet transform cannot improve the smooth effect and reserve image's precise details simultaneously; even false Gibbs phenomenon can be produced. This paper proposes a new image denoising method based on adaptive multiscale morphological edge detection beyond the above limitation. Firstly, the noisy image is decomposed by using one wavelet base. Then, the image edge is detected by using the adaptive multiscale morphological edge detection based on the wavelet decomposition. On this basis, wavelet coefficients belonging to the edge position are dealt with with the improved wavelet domain wiener filtering, and the others are dealt with with the improved Bayesian threshold and the improved threshold function. Finally, wavelet coefficients are inversely processed to obtain the denoised image. Experimental results show that this method can effectively remove the image noise without blurring edges and highlight the characteristics of image edge at the same time. The validation results of the denoised images with higher peak signal to noise ratio (PSNR) and structural similarity (SSIM) demonstrate their robust capability for real applications in the future.
\end{abstract}

\section{Introduction}

The image will be contaminated by random noise in the process of collection and transmission, which would inevitably lead to the degradation of the image quality in the subsequent process such as image compression and feature extraction. Hence, it is important to estimate the original image from the noisy image [1].

Image denoising is the basic problem of signal recovery in image process and is required to reduce or eliminate the noise of the observed images, while preserving the texture, corner, and edge details of the original image as much as possible is needed. Many scholars have devoted a lot of energy to the study of image denoising and put forward many effective methods over the past few decades. Wavelet analysis has good localization properties and multiresolution characteristics in either time domain or frequency domain, which makes it more effective to distinguish useful signal and noise. Therefore, wavelet has become a very effective method for the image denoising [2-10]. At present, there are mainly three kinds of wavelet denoising techniques to remove noise from image data. The first is to separate signal from noise through the singularity detection with wavelets [11-13]; the second is to reduce image noise through the wavelet coefficient thresholding method [14-16]; and the third is to reduce image noise through the wavelet domain Bayesian threshold criterion coefficient of shrinkage method [17-19]. Among them, wavelet coefficient thresholding method is the most widely used method for the image denoising because of its simpleness and effectiveness. The basic idea of wavelet coefficient thresholding method is derived from theory of Donoho. The general threshold denoising method based on orthogonal wavelet transform is initially presented by Donoho, which is based on the assumption that the complex noise reduction problem can be solved by simple coefficient process. However, the estimation of Donoho threshold does not have the adaptability for different scale spaces and will zero the wavelet coefficients in excess, which will lead to the loss in image details. So many scholars have proposed different scales of wavelet coefficients using the adaptive threshold to reduce noise, such as VisuShrink threshold, SureShrink threshold, and NormalShrink threshold. Although these algorithms can 
obtain better denoising effect to some extent, more details are eliminated so that the image quality is severely reduced; even false Gibbs phenomenon can be produced.

In this paper, we propose a new image denoising method based on adaptive multiscale morphological edge detection. Traditional or modern image edge detection method is accurate for the edge location of the nonnoisy image. However, the edge of the noisy image cannot be detected by it. In contrast, adaptive multiscale morphological edge detection method is able to locate the edge location of noisy image accurately. This is because the adaptive multiscale morphological edge detection method uses mathematical morphology theory based on the multistructuring elements and is constructed by using the improved morphological edge detection operator. Therefore, the adaptive multiscale morphological edge detection method can preserve more edge information by changing the size of structuring elements [20-22]. We decompose the noisy image by using wavelet firstly. Then, the edge of the image is detected via adaptive multiscale morphological edge detection according to the characteristics of the wavelet decomposition. On this basis, the wavelet coefficients belonging to the edge position are dealt with with the improved wavelet domain wiener filtering and the others are dealt with with the improved Bayesian threshold and the improved threshold function. Finally, wavelet coefficients are inversely processed to obtain the denoised image. The experimental results show that this method can not only remove the noise without blurring of edges and important characteristics of images but also highlight the characteristics of image edge compared with the existing methods. The denoised images obtain higher PSNR and higher SSIM; what is more, the denoising effect is better than the previous article [23-28]; hence, the method is of great application value.

\section{Adaptive Multiscale Morphological Edge Detection}

Suppose that the model of the image with Gauss white noise is as follows:

$$
y_{n}(i, j)=x(i, j)+n(i, j)
$$

where $(i, j)$ denotes the location of a pixel in the whole image space: $i=1,2, \ldots, M$, and $j=1,2, \ldots, N ; x(i, j)$ is the original image (not containing Gauss white noise) and its size is $M \times N ; n(i, j)$ is Gauss white noise.

The wavelet transform of the noisy image $y_{n}(i, j)$ is as follows:

$$
W_{y}=W_{x}+W_{n}
$$

where $W_{n}$ is the wavelet coefficient of the noise, $W_{x}$ is the wavelet coefficient of the original image, and $W_{y}$ is the wavelet coefficient of the noisy image.

Assume that $f(x, y)$ represents a gray-scale image and $b(x, y)$ is the given structuring element. $D_{f}$ and $D_{b}$ are the domains of definition of $f(x, y)$ and $b(x, y)$, respectively.
The dilation operation of $b(x, y)$ to the gray-scale image $f(x, y)$ is as follows:

$$
\begin{aligned}
& f \oplus b=\max \{f(s-x, t-y) \\
& \left.\quad+b(x, y) \mid(s-x),(t-y) \in D_{f} ;(x, y) \in D_{b}\right\} .
\end{aligned}
$$

The erosion operation of $b(x, y)$ to the gray-scale image $f(x, y)$ is as follows:

$$
\begin{aligned}
& f \Theta b=\min \{f(s+x, t+y) \\
& \left.\quad-b(x, y) \mid(s+x),(t+y) \in D_{f} ;(x, y) \in D_{b}\right\} .
\end{aligned}
$$

The opening operation of $b(x, y)$ to the gray-scale image $f(x, y)$ is as follows:

$$
f \circ b=(f \Theta b) \oplus b .
$$

The closing operation of $b(x, y)$ to the gray-scale image $f(x, y)$ is as follows:

$$
f \cdot b=(f \oplus b) \Theta b .
$$

Assume that $E(x, y)$ represents the image edge detection operator. Thus, an antinoise edge detection operator is obtained by using morphological dilation, erosion, opening, and closing operation.

Antinoise dilation operator is as follows:

$$
F_{1}=f \oplus b-f \cdot b .
$$

Antinoise erosion operator is as follows:

$$
F_{2}=f \circ b-f \Theta b .
$$

Antinoise dilation erosion operator is as follows:

$$
F_{3}=(f \circ b) \oplus b-(f \bullet b) \Theta b .
$$

Let

$$
\begin{aligned}
& F_{\text {min }}(x, y)=\min \left\{F_{1}(x, y), F_{2}(x, y), F_{3}(x, y)\right\} \\
& F_{\max }(x, y)=\max \left\{F_{1}(x, y), F_{2}(x, y), F_{3}(x, y)\right\} \\
& F_{\text {dec }}(x, y)=F_{\max }(x, y)-F_{\min }(x, y) .
\end{aligned}
$$

Since the edge of the image is weak and not continuous, we propose the following improved morphological edge detection operator to improve the image effect:

$$
E(x, y)=F_{3}(x, y)+\alpha F_{\mathrm{dec}}(x, y),
$$

where $0<\alpha<1$ is an adjustable parameter. Because the improved morphological edge detection operator can add image edge information, the discontinuity in image is reduced to some extent. When $\alpha$ becomes bigger, more edge information can be detected, but antinoise performance will drop. In order to strike a balance between more edge information and antinoise performance, usually $\alpha$ is between 0.3 and 0.6 . 
In order to reduce image noise and detect the detailed information of image edge in different directions, the choosing of structuring elements is a key factor in morphological image processing. The size and shape of structuring elements decide the final result of detected edges. Multiscale morphological edge detection is to use different sizes of structuring elements to extract edge characteristics of the image. Large scale structuring elements have good ability to remove the noise, but the detection result is rough. On the other hand, the ability to remove noise is weak for small scale structuring elements but can detect the edge details very well.

We use multiscale morphological edge detection to filter noise and keep image details simultaneously. Generally speaking, the $3 \times 3,5 \times 5$, and $7 \times 7$ windows are usually used, among which $3 \times 3$ window is the fastest, and its edge is the most exquisite [20]. In this paper, we choose six representative structuring elements as follows:

$$
\begin{aligned}
& b_{1}=\left[\begin{array}{lll}
0 & 1 & 0 \\
1 & 1 & 1 \\
0 & 1 & 0
\end{array}\right] \\
& b_{2}=\left[\begin{array}{lll}
0 & 1 & 0 \\
1 & 1 & 0 \\
0 & 0 & 0
\end{array}\right] \\
& b_{3}=\left[\begin{array}{lll}
0 & 0 & 0 \\
0 & 1 & 1 \\
0 & 1 & 0
\end{array}\right] \\
& b_{4}=\left[\begin{array}{lll}
0 & 0 & 0 \\
1 & 1 & 0 \\
0 & 1 & 0
\end{array}\right] \\
& b_{5}=\left[\begin{array}{lll}
0 & 1 & 0 \\
0 & 1 & 1 \\
0 & 0 & 0
\end{array}\right] \\
& b_{6}=\left[\begin{array}{lll}
1 & 0 & 1 \\
0 & 1 & 0 \\
1 & 0 & 1
\end{array}\right],
\end{aligned}
$$

where $b_{i}$ is $3 \times 3$ square window: $i=1,2, \ldots, 6$.

Multiscale structuring elements are defined as $n b=b \oplus$ $b \oplus \cdots \oplus b$, where $n$ is scale parameter (a positive integer) and $b$ is the given structuring element. In addition, $n b$ indicates that the large scale structuring elements are obtained by the dilation of small scale elements.

The edge detection operators $E_{i}(x, y)(i=1,2, \ldots, 6)$ of six representative structuring elements above can be obtained by (13). Because different structuring elements have different adaptability to detect image edge, in order to preserve more edge information and make the edge more smooth, we first give different weight coefficients $\beta_{n i}$ to $E_{i}(x, y)$. Because performance of antinoise of the large scale structuring elements is more obvious but that of small scale structuring elements is relatively weak, it is necessary to utilize this feature to distinguish the edge of image. So we introduce the adaptive coefficient $\beta_{n i}$, which can better filter out the pseudoedge at large scale and take the advantages of multiscale edge detection and better detect the edge of the image. Then sum up $E_{i}(x, y)$; that is, $E_{n b}(x, y)=\bigcup_{i=1}^{6} \beta_{n i} E_{i}(x, y)$, where $n$ represents scale. Image edge can be detected using $E_{n b}(x, y)$. By changing the scale $n$ of the structuring elements, the image edge information is obtained. The new edge is as follows:

$$
f^{\prime}(x, y)=\sum_{n=l_{1}}^{l_{2}} E_{n b}(x, y)
$$

where $\left[l_{1}, l_{2}\right]$ is the range of scale $n$.

The weight coefficient $\beta_{n i}$ is calculated as follows:

(1) Get the mean filtered image under different scales structuring elements:

$$
f_{n}(x, y)=\frac{f(f \circ n b \cdot n b+f \cdot n b \circ n b)}{2} .
$$

(2) Calculate the standard deviation under different scales: $\Delta_{n}=\left|f-f_{n}\right|$.

(3) Based on the fact that the weight coefficients $\beta_{n i}$ are inversely proportional to the standard deviation $\Delta_{n}$, that is to say, large scale weight coefficients are bigger and small scale weight coefficients are smaller, we have

$$
\beta_{n i}=\frac{k_{i} \Delta_{l_{2}-n}}{\sum_{n=l_{1}}^{l_{2}} \Delta_{n}},
$$

where $k_{i}$ represents adjustable parameters related with $b_{i}: i=$ $1,2, \ldots, 6$.

\section{Improved Threshold Denoising}

Bayesian threshold is derived through the statistical theory of Bayes. For a given high frequency subband, the traditional threshold value is

$$
\lambda=\frac{\sigma_{\text {Noise }}^{2}}{\sigma_{\text {Image }}},
$$

where $\sigma_{\text {Noise }}$ and $\sigma_{\text {Image }}$ are the estimation for the noise variance and the standard deviation of the image on the subband, respectively.

The noise variance is estimated as follows:

$$
\sigma_{\text {Noise }}=\frac{\operatorname{median}\left(\left|Y_{i, j}\right|\right)}{0.6745},
$$

where $Y_{i, j}$ is the wavelet coefficient of subband $\mathrm{HH}_{1}$ of wavelet decomposition and median $(\cdot)$ is the median function. 
The standard deviation of the image is estimated as follows:

$$
\sigma_{\text {Image }}^{2}=\left.\left|\frac{1}{I \times J} \sum_{i, j}\right| W_{I \times J}\left(s_{i, j}\right)\right|^{2}-\left(\sigma_{\text {Noise }}\right)^{2} \mid,
$$

where $W_{I \times J}$ presents a template with a size of $I \times J$; $W_{I \times J}\left(s_{i, j}\right)$ present coefficient values of the image in the template. However, the distribution characteristics of the noise during the wavelet decomposition are not taken into account in (18).

Here, we introduce an adaptive coefficient $\eta$, which changes with wavelet coefficients adaptively. Therefore, the adaptive threshold is as follows:

$$
T=\eta \lambda
$$

where $\eta=1 / \log (K+1) ; K$ is the number of decomposition levels. We improve the traditional threshold in (18) so that the improved Bayesian threshold in (21) can change with the number of decomposition levels $K$ adaptively. When the decomposition level $K$ increases, the threshold becomes smaller, which is in line with distribution characteristics of the noise decomposed by wavelet. By this way, the noise is removed to the maximum extent possible, but the wavelet coefficients of the original image are not affected.

Next, we propose the improved threshold function as follows:

$$
f \begin{cases}\operatorname{sgn}(y(i, j)) p \sqrt{|y(i, j)|^{p}-\left(\frac{T}{|y(i, j)|}\right)^{p} T^{p}} & |y(i, j)|>T \\ 0 & |y(i, j)| \leq T\end{cases}
$$

where $p$ can be adjusted according to the wavelet coefficients $y(i, j)$ and usually $p=20 r$. Here, $r$ is the percentage of the wavelet coefficients $y(i, j)$ and is greater than $2 T$. The improved threshold function $f$ can automatically change with wavelet coefficients, which can effectively overcome the defects of hard threshold function and soft threshold function.

\section{Improved Wavelet Domain Wiener Filtering}

Wiener filter is a locally adaptive linear filter using observation window. Assuming that the size of the observation window is $m \times n$, usually take $m=n$. The following steps are locally adaptive wiener filtering in wavelet domain and can obtain the denoised image.

(1) Calculate the variance of the wavelet coefficients of the noisy image with observation window units:

$$
q(i, j)=\widehat{\sigma}_{y(i, j)}^{2}=\frac{1}{M} \sum y^{2}(i, j)
$$

where $y(i, j)$ is the wavelet coefficient; $M=m \bullet n$.

(2) Calculate the variance of the wavelet coefficients of the original image without noise:

$$
\widehat{\sigma}_{x(i, j)}^{2}=\max \left(\widehat{\sigma}_{y(i, j)}^{2}-\sigma_{\text {Noise }}^{2}, 0\right),
$$

where $\sigma_{\text {Noise }}$ is the noise variance and can be calculated by (19).
(3) Process wavelet coefficients as follows:

$$
\widehat{x}(i, j)=\frac{\widehat{\sigma}_{x(i, j)}^{2}}{\left(\widehat{\sigma}_{x(i, j)}^{2}+\sigma_{\text {Noise }}^{2}\right)} \cdot y(i, j) .
$$

In the following, we will improve wavelet domain wiener filtering through adding threshold processing before step (3). The steps added are as follows:

(1) Select the appropriate wavelet "coif3" to decompose the noisy image and the decomposition level is $K=4$.

(2) Estimate the noise variance $\sigma_{\text {Noise }}^{2}$ from the high frequency subband $\mathrm{HH}_{1}$.

(3) Calculate parameter $c$ of each scale in order to further improve the adaptability of threshold.

$$
c=\sqrt{\frac{L_{k}}{L} \log \left(\frac{L_{k}}{K}\right)},
$$

where $L_{k}$ is the length of $k$ th subband, $L$ is the length of the noisy image, $k=1,2, \ldots, K$, and $K$ is the number of decomposition levels. It is obvious that the parameter $c$ will change adaptively with $K$.

(4) The wavelet coefficients are processed as follows:

$$
y(i, j)= \begin{cases}y(i, j) & q(i, j) \geq c \sigma_{\text {Noise }}^{2} \\ 0 & q(i, j)<c \sigma_{\text {Noise }}^{2}\end{cases}
$$

Parameter $c$ in (26) is related to SNR (signal to noise ratio). The bigger SNR becomes, the bigger parameter $c$ becomes, the more details are retained, and the clearer the image becomes. In addition, the improved wavelet domain wiener filtering implements the minimum variance estimation. What is more, this treatment is consistent with the visual characteristics of human and the denoised image has better visual effects.

\section{Denoising Method Proposed in This Paper}

It is notable that many problems still exist in the threshold denoising. Worst of all is the fact that too much detail is eliminated, so that the image quality is severely reduced; even pseudo-Gibbs phenomenon is produced.

Combing improved Bayesian threshold with wavelet domain wiener filtering, a new method based on adaptive multiscale morphological edge detection is proposed to solve the threshold denoising problems above. Steps are as follows.

Step 1. Use the wavelet base "sym3" to decompose the noisy image into four layers.

Step 2. For each group of wavelet coefficients, apply adaptive multiscale morphological edge detection method to detect wavelet coefficients belonging to the edge of the image.

Step 3. Deal with wavelet coefficients belonging to the edge through improved wavelet domain wiener filtering, and window size of $5 \times 5$ is chosen for each group. 
Step 4. Deal with wavelet coefficients not belonging to the edge with improved threshold denoising.

Step 5. Inversely process wavelet coefficients through the wavelet base "sym3" for each group to get the final denoised image.

The proposed method in this paper has the following characteristics. Firstly, this method based on adaptive multiscale morphological edge detection can not only detect edge details but also protect and highlight them. So this method has higher PSNR and SSIM. Secondly, the traditional Bayesian threshold is improved by adding adaptive coefficient $\eta=1 / \log (K+1)$, which can change with the number of decomposition levels $K$ adaptively. To be more precise, when the decomposition level $K$ increases, the threshold becomes smaller. This feature is in line with distribution characteristics of the noise decomposed by wavelet. By this way, the noise is removed to the maximum extent possible, but the wavelet coefficients of the original image are not affected. Finally, wavelet domain wiener filtering is improved by adding parameter $c$ in order to further enhance the adaptability of threshold, which can retain edge features and increase clarity of the image. Thus, the proposed method has better visual effects and can provide better visual performance.

\section{Image Denoising Evaluation}

At present, there are two ways to evaluate the quality of the denoised image: subjective evaluation and objective evaluation. Subjective evaluation is to evaluate the denoising effect from the qualitative point of view. On the contrary, objective evaluation is to evaluate the denoising effect from the quantitative point of view. However, there are no uniform evaluation criteria to evaluate the denoising effect until now. PSNR and SSIM are adopted to evaluate the results of denoised image in $[29,30]$.

Assuming that $f(i, j)$ is the gray value of the original image without noise and $D(i, j)$ is the gray value of the denoised image, the size of the image is $M \times N$.

PSNR is defined as follows:

$$
\begin{aligned}
\text { MSE } & =\frac{1}{M \cdot N} \sum_{i=1}^{M} \sum_{j=1}^{N}[f(i, j)-D(i, j)] \\
\text { PSNR } & =10 \log _{10}\left[\frac{255^{2}}{\mathrm{MSE}}\right] .
\end{aligned}
$$

The smaller MSE value is, the bigger PSNR value is. It means that the difference between the original image and the denoised image is small, and the denoising effect is better.

SSIM is a kind of image quality evaluation index based on structural similarity. Assuming that the human eye is suitable for extracting the structural information from the perspective of the visual field, we have

$$
\operatorname{SSIM}=\frac{\left(2 u_{1} u_{2}+c_{1}\right)}{\left(u_{1}^{2}+u_{2}^{2}+c_{1}\right)} \frac{\left(2 \sigma_{12}+c_{2}\right)}{\left(\sigma_{1}^{2}+\sigma_{2}^{2}+c_{2}\right)},
$$

where $u_{1}$ and $u_{2}$ are the pixel means before and after the treatment, respectively. $\sigma_{k}$ is the pixel variance, $\sigma_{12}$ is the pixel covariance, and $c_{k}$ is constant: $k=1,2$. In this paper, we take $c_{1}=(0.01 \times 255)^{2}$ and $c_{2}=(0.03 \times 255)^{2}$. The range of SSIM is $[0,1]$ and its maximum value is 1 . If SSIM is closer to 1 , it indicates that the denoised image is more similar to the original image. This is to say that more edge details of the original image can be retained.

\section{Simulation Experiments}

In this section, three groups of experiments are provided to illustrate the effectiveness of the proposed method in this paper.

Figure 1 is an example of remote sensing image. The denoised image is Aerial image with the size of $512 \times 512$ pixels. We compare the denoising results with articles [23-28] from the qualitative and quantitative aspects. The denoised images are shown in Figure 1 (the noise standard deviation $\sigma_{n}=30$ ), and the comparison results of denoised index PSNR and SSIM are shown in Table 1.

In Figure 1, the image processed by [23, 24, 27] or [28] is more blurred because it loses much edge information. The image processed by [25] or [26] includes much noise which destroys the clarity of the image. On the contrary, the denoising effect of the new method proposed in this paper is more significant and can preserve more edge information than the others.

From Table 1, we find that the new method has better performance than the others for Aerial image. The results of experiments show the high effectiveness of the proposed method in this paper for remote sensing image, no matter from the subjective or objective aspects.

Figure 2 is an example of Lena, Barbara, and Boat image with the size of $512 \times 512$ pixels. We compare denoising results with articles [23-28] from qualitative and quantitative aspects. The denoised images are shown in Figure 2 (the noise standard deviation $\sigma_{n}=30$ ), and the comparison results of denoised index PSNR and SSIM are shown in Table 2.

In Figure 2, the new method highlights the features of the edge and retains most of the edge information. So the denoised edge details are more obvious and closer to the original image. Furthermore, we magnify the details to show the subtle differences between different denoised images. Because image denoising method based on adaptive multiscale morphological edge detection can detect the image edges with different direction structuring elements and each structuring element can detect an image edge, the ideal image edge with detailed character in Figure $2(\mathrm{k})$ can be obtained by combining different orientations.

In addition, from Table 2, we find that PSNR and SSIM of the reconstructed image obtained by the new method are highest. It is shown that the new method has strong denoising ability no matter in the low SNR or in the high SNR. In other words, the denoised images processed by the new method have higher peak signal to noise ratio and mean structural similarity.

Figure 3 is an example of infrared image. The denoised image is infrared image of Cassiopeia A with the size of $640 \times$ 480 pixels. We compare denoising results with articles [2328] from qualitative and quantitative aspects. The denoised 


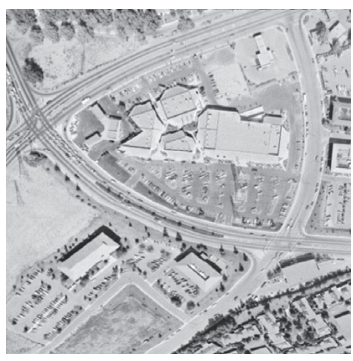

(a) The original image

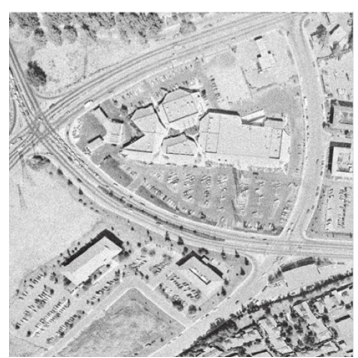

(c) Article [23]

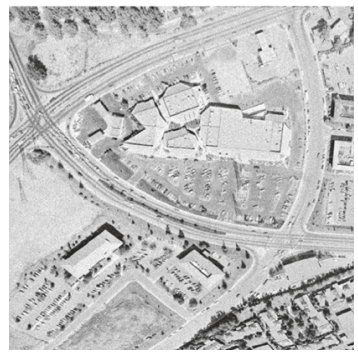

(e) Article [25]

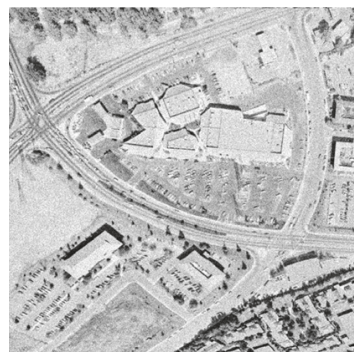

(g) Article [27]

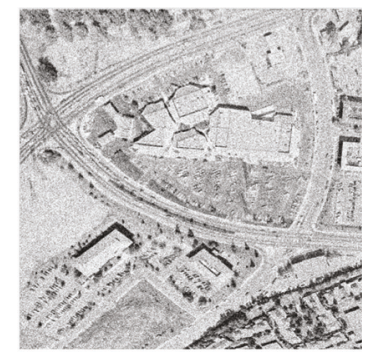

(b) The noisy image

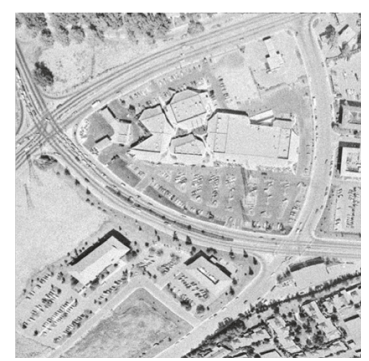

(d) Article [24]

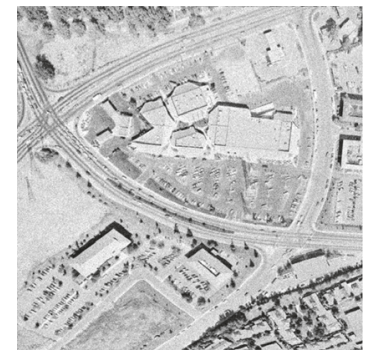

(f) Article [26]

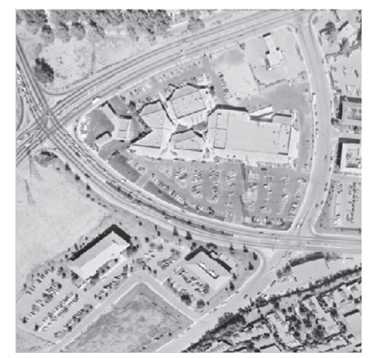

(h) Article [28]

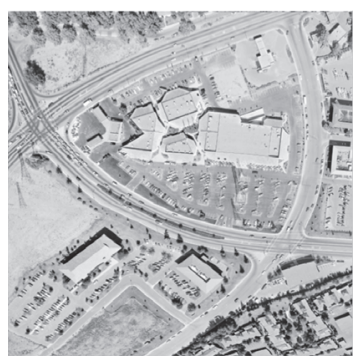

(i) The new method

FIGURE 1: Comparison of different image denoising methods for Aerial image. 


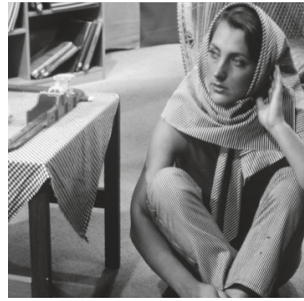

(a) The original image

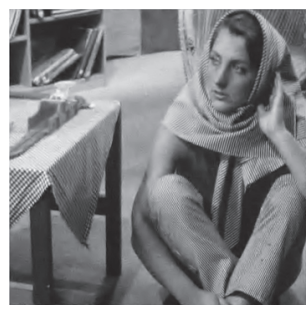

(c) Article [23]

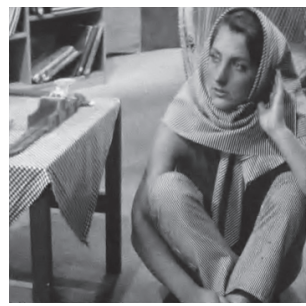

(e) Article [25]

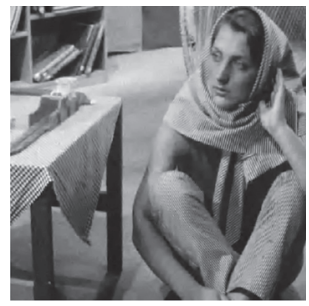

(g) Article [27]

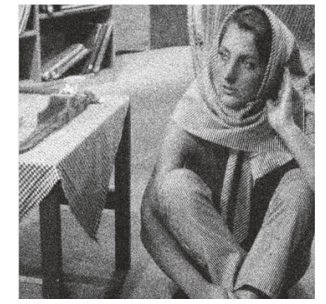

(b) The noisy image

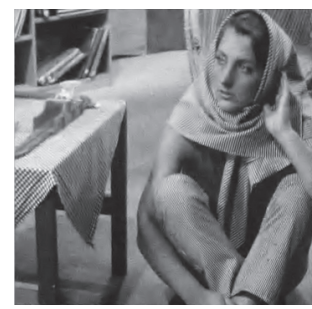

(d) Article [24]

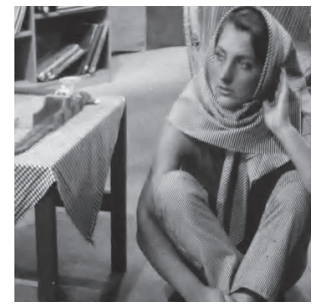

(f) Article [26]

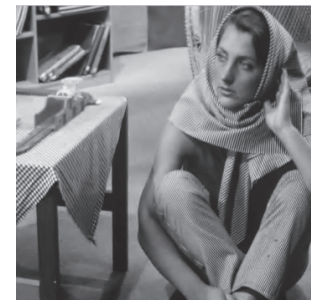

(h) Article [28]

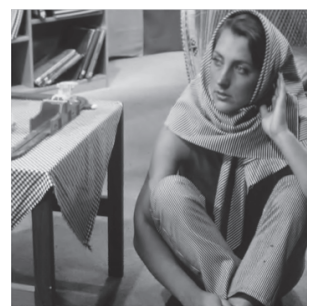

(i) The new method

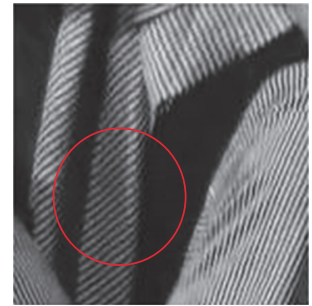

(j) Magnified images cropped from $(\mathrm{h})$

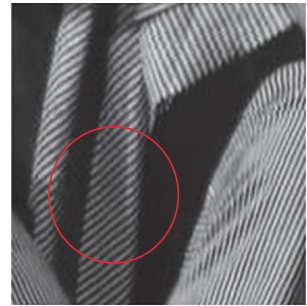

(k) Magnified images cropped from (i)

FIGURE 2: Comparison of denoising methods for image Barbara. 


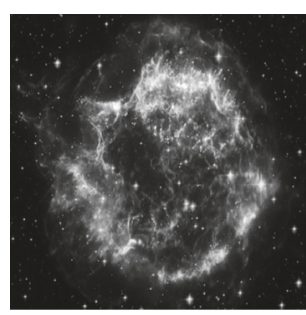

(a) The original image

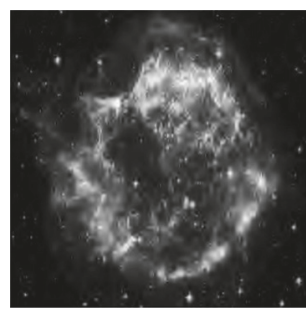

(c) Article [23]

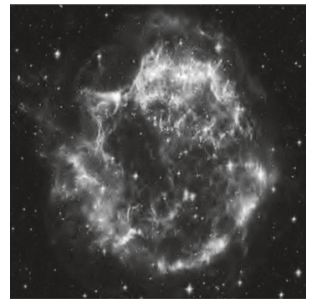

(e) Article [25]

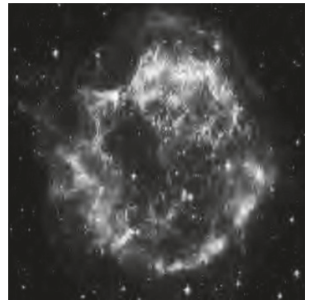

(g) Article [27]

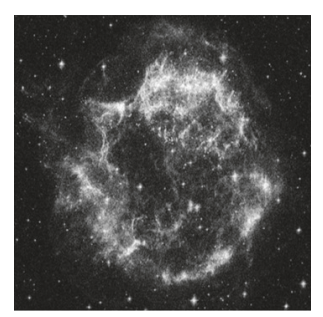

(b) The noisy image

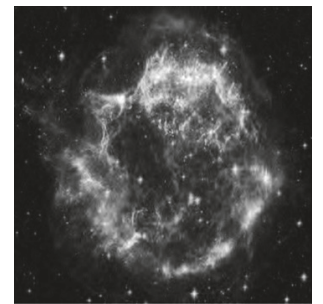

(d) Article [24]

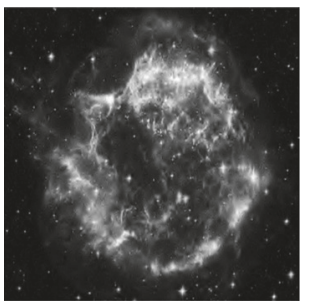

(f) Article [26]

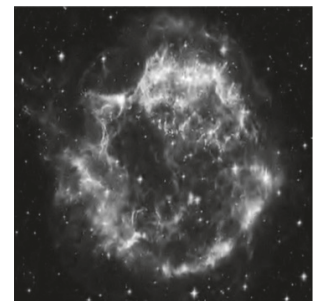

(h) Article [28]

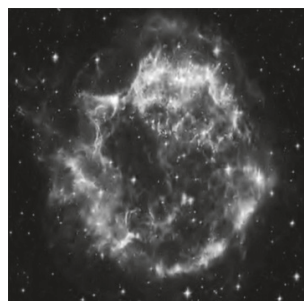

(i) The new method
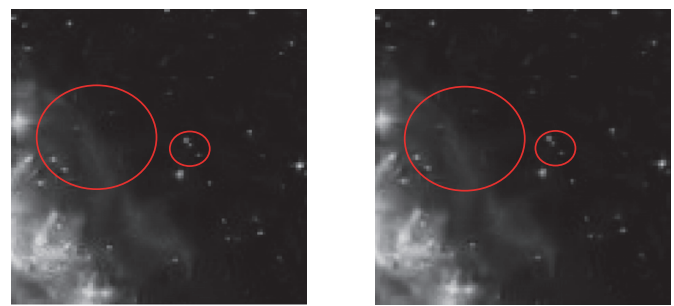

(j) Magnified images cropped from (h)

(k) Magnified images cropped from (i)

FIGURE 3: Comparison of different infrared image denoising methods for Cassiopeia A. 
TABLE 1: Comparison of different denoising methods on PSNR and SSIM for Aerial image.

\begin{tabular}{|c|c|c|c|c|c|c|}
\hline \multirow{2}{*}{ Denoising method } & \multicolumn{2}{|c|}{$\sigma_{n}=10$} & \multicolumn{2}{|c|}{$\sigma_{n}=20$} & \multicolumn{2}{|c|}{$\sigma_{n}=30$} \\
\hline & PSNR & SSIM & PSNR & SSIM & PSNR & SSIM \\
\hline Noisy image & 28.1428 & 0.6488 & 22.3143 & 0.4013 & 18.5941 & 0.2612 \\
\hline Article [23] & 31.5413 & 0.8224 & 26.8412 & 0.7133 & 24.8298 & 0.6328 \\
\hline Article [24] & 31.6817 & 0.8341 & 27.1353 & 0.7262 & 24.9526 & 0.6581 \\
\hline Article [25] & 31.8256 & 0.8536 & 27.3763 & 0.7486 & 25.1833 & 0.6739 \\
\hline Article [26] & 31.1601 & 0.8110 & 27.0601 & 0.7021 & 24.1912 & 0.6525 \\
\hline Article [27] & 31.6325 & 0.8882 & 27.8442 & 0.7858 & 25.6135 & 0.7083 \\
\hline Article [28] & 31.8812 & 0.8991 & 27.9914 & 0.8164 & 25.6002 & 0.7213 \\
\hline The new method & 32.0131 & 0.9189 & 28.0675 & 0.8343 & 25.9070 & 0.7553 \\
\hline
\end{tabular}

TABLE 2: Comparison of different denoising methods on PSNR and SSIM for images.

\begin{tabular}{lccccccccc}
\hline Denoising method & \multicolumn{3}{c}{ Lena } & & \multicolumn{3}{c}{ Barbara } \\
& $\sigma_{n}=10$ & $\sigma_{n}=20$ & $\sigma_{n}=30$ & $\sigma_{n}=10$ & $\sigma_{n}=20$ & $\sigma_{n}=30$ & $\sigma_{n}=10$ & $\sigma_{n}=20$ & $\sigma_{n}=30$ \\
\hline \multirow{2}{*}{ Noisy image } & 28.18 & 22.15 & 18.67 & 28.12 & 22.12 & 18.66 & 28.14 & 22.16 & 18.62 \\
& 0.5646 & 0.3398 & 0.2333 & 0.7036 & 0.4945 & 0.3673 & 0.6072 & 0.3992 & 0.2888 \\
Article [23] & 34.53 & 31.56 & 29.88 & 32.81 & 28.68 & 26.79 & 32.93 & 29.75 & 27.68 \\
& 0.8894 & 0.8282 & 0.7683 & 0.8846 & 0.8318 & 0.7632 & 0.8498 & 0.7746 & 0.7226 \\
Article [24] & 34.27 & 31.07 & 28.96 & 31.96 & 28.16 & 26.11 & 32.37 & 29.11 & 27.31 \\
& 0.8798 & 0.8175 & 0.7618 & 0.8503 & 0.7938 & 0.7323 & 0.8324 & 0.7763 & 0.7105 \\
Article [25] & 33.37 & 29.63 & 27.35 & 32.76 & 28.29 & 26.57 & 32.03 & 28.02 & 26.29 \\
& 0.8878 & 0.8013 & 0.7856 & 0.8996 & 0.8489 & 0.7811 & 0.8768 & 0.7863 & 0.6802 \\
Article [26] & 34.98 & 31.73 & 29.62 & 33.01 & 29.18 & 27.01 & 33.11 & 29.77 & 27.74 \\
& 0.9035 & 0.8494 & 0.8066 & 0.9161 & 0.8528 & 0.7837 & 0.8573 & 0.7953 & 0.7106 \\
Article [27] & 33.98 & 30.86 & 28.68 & 31.76 & 27.37 & 26.31 & 32.58 & 29.32 & 27.72 \\
& 0.8469 & 0.7484 & 0.6586 & 0.8678 & 0.7723 & 0.7426 & 0.8302 & 0.7563 & 0.6752 \\
Article [28] & 34.81 & 31.28 & 30.03 & 31.62 & 29.02 & 26.81 & 32.66 & 29.18 & 28.12 \\
& 0.8990 & 0.8545 & 0.8203 & 0.9074 & 0.8331 & 0.7780 & 0.8387 & 0.7619 & 0.7129 \\
The new method & $\mathbf{3 5 . 2 1}$ & $\mathbf{3 1 . 7 6}$ & $\mathbf{3 0 . 0 8}$ & $\mathbf{3 3 . 0 2}$ & $\mathbf{2 9 . 1 9}$ & $\mathbf{2 7 . 2 1}$ & $\mathbf{3 3 . 4 8}$ & $\mathbf{3 0 . 1 8}$ & $\mathbf{2 8 . 3 4}$ \\
& $\mathbf{0 . 9 0 7 0}$ & $\mathbf{0 . 8 5 5 8}$ & $\mathbf{0 . 8 2 1 2}$ & $\mathbf{0 . 9 2 1 8}$ & $\mathbf{0 . 8 5 3 2}$ & $\mathbf{0 . 7 9 2 1}$ & $\mathbf{0 . 8 7 8 8}$ & $\mathbf{0 . 7 9 7 6}$ & $\mathbf{0 . 7 4 0 8}$ \\
\hline
\end{tabular}

images are shown in Figure 3 (the noise standard deviation $\sigma_{n}=30$ ), and the comparison results of denoised index PSNR and SSIM are shown in Table 3.

In Figure 3, the image processed by [23, 24] or [27] is more blurred because it loses much edge information. The image processed by [25] or [26] includes much noise which destroys the clarity of the image. The denoising effect of [28] is more close to our proposed method and can preserve more edge information than the others. A Further comparison between Figures 3(j) and 3(k) illustrates that the denoised edge details are more obvious using the proposed method in this paper.

From Table 3, we find that the new method has better performance than the others for the infrared image.

The proposed method in this paper is dependent on parameter $\alpha$ in (13). Linear parameter $\alpha$ is the weighting value of $F_{\mathrm{dec}}(x, y)$ in (12) to adjust edge detection operator, whose sensitivity is decided by $F_{\mathrm{dec}}(x, y)$. When $\alpha$ becomes bigger, more edge information can be detected, but antinoise performance will drop. Through repeated experiments, the denoising effect is ideal when $\alpha$ is between 0.3 and 0.6 . In this paper, we set $\alpha=0.5$. Figure 4 shows the PSNR of Aerial image, Barbara, and Cassiopeia A for parameter $\alpha \in[0,1]$.
At last, we note down the computational cost of different denoising methods. We test the algorithms to process one $512 \times 512$ image on a Windows machine with Intel(R) Celeron G550 @ 2.60 GHz CPU and 4.00 GB RAM. Results are illustrated in Table 4.

In [27], most of the runtime latency was caused by the reading of coefficients back from GPU's framebuffer and the sorting operation on CPU, and the runtime of these two tasks dramatically increases along with the image size. In [26], since the BRISQUE approach requires a training procedure to calibrate the regressor module, the computational complexity is high. Although the method in [28] dividing the image into three segments is more close to our proposed method, from the above comparisons, it can be concluded that our proposed method allows an efficient denoising algorithm with relatively low complexity.

The computation times have been averaged over twenty runs.

\section{Conclusion}

In this paper, we propose a new method to remove Gaussian noise from digital images. Based on adaptive multiscale 
TABLE 3: Comparison of different denoising methods on PSNR and SSIM for Cassiopeia A.

\begin{tabular}{|c|c|c|c|c|c|c|}
\hline \multirow{2}{*}{ Denoising method } & \multicolumn{2}{|c|}{$\sigma_{n}=10$} & \multicolumn{2}{|c|}{$\sigma_{n}=20$} & \multicolumn{2}{|c|}{$\sigma_{n}=30$} \\
\hline & PSNR & SSIM & PSNR & SSIM & PSNR & SSIM \\
\hline Noisy image & 28.1328 & 0.6297 & 22.1122 & 0.3594 & 18.5904 & 0.2291 \\
\hline Article [23] & 31.9312 & 0.8286 & 28.1201 & 0.8035 & 27.9811 & 0.6882 \\
\hline Article [24] & 31.1877 & 0.8415 & 28.1838 & 0.7762 & 26.8825 & 0.6673 \\
\hline Article [25] & 30.0256 & 0.7866 & 27.7673 & 0.7544 & 25.7336 & 0.6694 \\
\hline Article [26] & 32.3656 & 0.8810 & 30.1614 & 0.8201 & 28.8912 & 0.7325 \\
\hline Article [27] & 30.5267 & 0.8092 & 28.4142 & 0.7785 & 26.3635 & 0.6738 \\
\hline Article [28] & 32.5267 & 0.8819 & 30.2484 & 0.8234 & 28.6222 & 0.7363 \\
\hline The new method & 33.9213 & 0.8989 & 31.0765 & 0.8266 & 29.0733 & 0.7458 \\
\hline
\end{tabular}

TABLE 4: Comparison of different denoising methods on computational burdens.

\begin{tabular}{lccccccc}
\hline Method & Article [23] & Article [24] & Article [25] & Article [26] & Article [27] & Article [28] & The new method \\
\hline Unit of time (s) & 88 & 1.7782 & 1.5506 & 1.3011 & 1.1112 & 0.9382 & 0.9532 \\
\hline
\end{tabular}

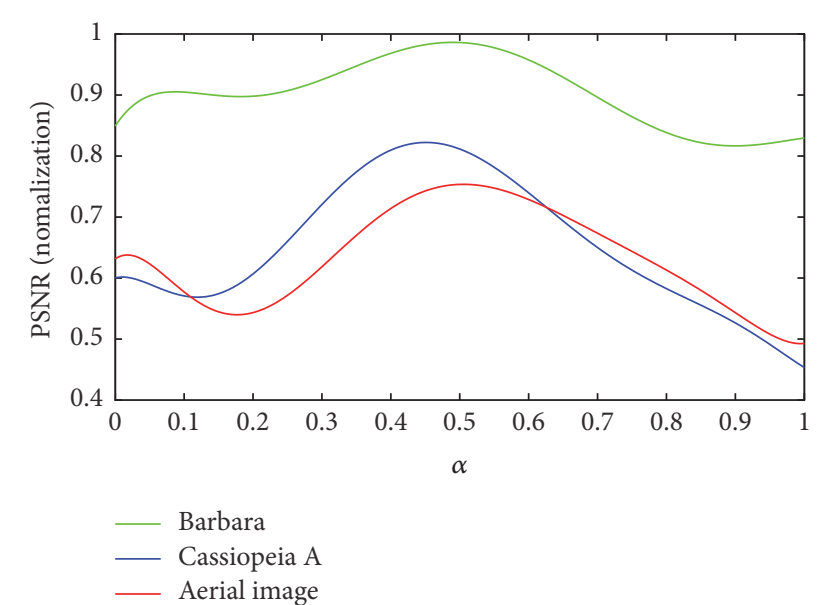

FIgure 4: The PSNR of Aerial image, Barbara, and Cassiopeia A for parameter $\alpha \in[0,1]$.

morphological edge detection combining improved Bayesian threshold and wiener filtering, the method proposed in this paper not only can remove the noise from remote sensing image but also can remove the noise from general image. It is able to retain the edge details of the image and highlight features of the edge. The simulation experiments show that the denoised images have higher peak signal to noise ratio and mean structural similarity and are of great application value.

\section{Conflicts of Interest}

The authors declare that they have no conflicts of interest.

\section{Acknowledgments}

This work is supported by the National Natural Science Foundation of China (61374124, 6147306, and 61627809).

\section{References}

[1] Y. Shi, X. Yang, and Y. Guo, "Translation invariant directional framelet transform combined with Gabor filters for image denoising," IEEE Transactions on Image Processing, vol. 23, no. 1, pp. 44-55, 2014.

[2] H. Zhong, K. Ma, and Y. Zhou, "Modified BM3D algorithm for image denoising using nonlocal centralization prior," Signal Processing, vol. 106, pp. 342-347, 2015.

[3] X.-C. Feng, L. Luo, X.-X. Jia, and W.-W. Wang, "A divideand-conquer stochastic alterable direction image denoising method," Signal Processing, vol. 108, pp. 90-101, 2015.

[4] P. Sharma, K. Khan, and K. Ahmad, "Image denoising using local contrast and adaptive mean in wavelet transform domain," International Journal of Wavelets, Multiresolution and Information Processing, vol. 12, no. 6, Article ID 1450038, 15 pages, 2014.

[5] S. Kim, W. Kang, E. Lee, and J. Paik, "Wavelet-domain color image enhancement using filtered directional bases and frequency-adaptive shrinkage," IEEE Transactions on Consumer Electronics, vol. 56, no. 2, pp. 1063-1070, 2010.

[6] R. Yan, L. Shao, and Y. Liu, "Nonlocal hierarchical dictionary learning using wavelets for image denoising," IEEE Transactions on Image Processing, vol. 22, no. 12, pp. 4689-4698, 2013.

[7] P. R. Hill, A. M. Achim, D. R. Bull, and M. E. Al-Mualla, "Dualtree complex wavelet coefficient magnitude modelling using the bivariate Cauchy-Rayleigh distribution for image denoising," Signal Processing, vol. 105, pp. 464-472, 2014.

[8] N. Renard, S. Bourennane, and J. Blanc-Talon, "Denoising and dimensionality reduction using multilinear tools for hyperspectral images," IEEE Geoscience and Remote Sensing Letters, vol. 5, no. 2, pp. 138-142, 2008.

[9] D. Cho and T. D. Bui, "Fast image enhancement in compressed wavelet domain," Signal Processing, vol. 98, pp. 295-307, 2014.

[10] Y. Xu, J. B. Weaver, D. M. Healy, and J. Lu, "Wavelet transform domain filters: a spatially selective noise filtration technique," IEEE Transactions on Image Processing, vol. 3, no. 6, pp. 747-758, 1994.

[11] V. Bruni and D. Vitulano, "Wavelet-based signal de-noising via simple singularities approximation," Signal Processing, vol. 86, no. 4, pp. 859-876, 2006. 
[12] S. Yin, L. Cao, Y. Ling, and G. Jin, "Image denoising with anisotropic bivariate shrinkage," Signal Processing, vol. 91, no. 8, pp. 2078-2090, 2011.

[13] S. Mallat and W. L. Hwang, "Singularity detection and processing with wavelets," IEEE Transactions on Information Theory, vol. 38, no. 2, pp. 617-643, 1992.

[14] D. Min, Z. Jiuwen, and M. Yide, "Image denoising via bivariate shrinkage function based on a new structure of dual contourlet transform," Signal Processing, vol. 109, no. 4, pp. 25-37, 2015.

[15] R. Rubinstein and M. Elad, "Dictionary learning for analysissynthesis thresholding," IEEE Transactions on Signal Processing, vol. 62, no. 22, pp. 5962-5972, 2014.

[16] D. L. Donoho, "De-noising by soft-thresholding," IEEE Transactions on Information Theory, vol. 41, no. 3, pp. 613-627, 1995.

[17] D. H. P. Salvadeo, N. D. A. Mascarenhas, and A. L. M. Levada, "Nonlocal Markovian models for image denoising," Journal of Electronic Imaging, vol. 25, no. 1, Article ID 013003, 2016.

[18] J. Ho and W.-L. Hwang, "Wavelet Bayesian network image denoising," IEEE Transactions on Image Processing, vol. 22, no. 4, pp. 1277-1290, 2013.

[19] S. G. Chang, B. Yu, and M. Vetterli, "Adaptive wavelet thresholding for image denoising and compression," IEEE Transactions on Image Processing, vol. 9, no. 9, pp. 1532-1546, 2000.

[20] L. J. Huang and H. Zhou, "A method of multi structure elements based on morphological image edge detection," Microelectronics and Computer, vol. 26, no. 8, pp. 76-79, 2009.

[21] Z. J. Xiang and P. J. Ramadge, "Edge-preserving image regularization based on morphological wavelets and dyadic trees," IEEE Transactions on Image Processing, vol. 21, no. 4, pp. 15481560, 2012.

[22] C. Ni, Q. Li, and L. Z. Xia, "A novel method of infrared image denoising and edge enhancement," Signal Processing, vol. 88, no. 6, pp. 1606-1614, 2008.

[23] L. Srinivasan, Y. Rakvongthai, and S. Oraintara, "Microarray image denoising using complex gaussian scale mixtures of complex wavelets," IEEE Journal of Biomedical and Health Informatics, vol. 18, no. 4, pp. 1423-1430, 2014.

[24] G. Shikkenawis, S. K. Mitra, and A. Rajwade, "Image denoising using orthogonal locality preserving projections," Journal of Electronic Imaging, vol. 24, no. 4, Article ID 43018, 2015.

[25] C.-A. Deledalle, L. Denis, F. Tupin, A. Reigber, and M. Jager, "NL-SAR: a unified nonlocal framework for resolutionpreserving (Pol)(In)SAR denoising," IEEE Transactions on Geoscience and Remote Sensing, vol. 53, no. 4, pp. 2021-2038, 2015.

[26] A. Mittal, A. K. Moorthy, and A. Bovik, "No-reference image quality assessment in the spatial domain," IEEE Transactions on Image Processing, vol. 21, no. 12, pp. 4695-4708, 2012.

[27] Y. Su and Z. Xu, "Parallel implementation of wavelet-based image denoising on programmable PC-grade graphics hardware," Signal Processing, vol. 90, no. 8, pp. 2396-2411, 2010.

[28] H. K. Rafsanjani, M. H. Sedaaghi, and S. Saryazdi, "Efficient diffusion coefficient for image denoising," Computers \& Mathematics with Applications, vol. 72, no. 4, pp. 893-903, 2016.

[29] L. Gao, G. Wang, and J. Liu, "Image denoising based on edge detection and prethresholding Wiener filtering of multiwavelets fusion," International Journal of Wavelets, Multiresolution and Information Processing, vol. 13, no. 5, Article ID 1550031, 15 pages, 2015.
[30] S. P. Maity, A. Phadikar, and M. K. Kundu, "Image error concealment based on QIM data hiding in dual-tree complex wavelets," International Journal of Wavelets, Multiresolution and Information Processing, vol. 10, no. 2, Article ID 1250016, 30 pages, 2012. 


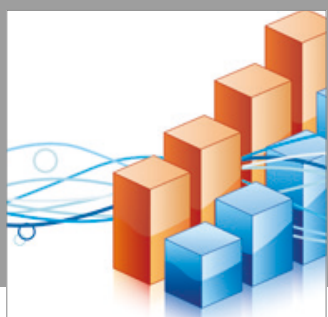

Advances in

Operations Research

vatersals

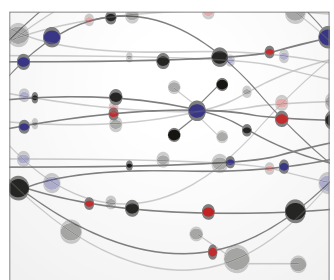

\section{The Scientific} World Journal
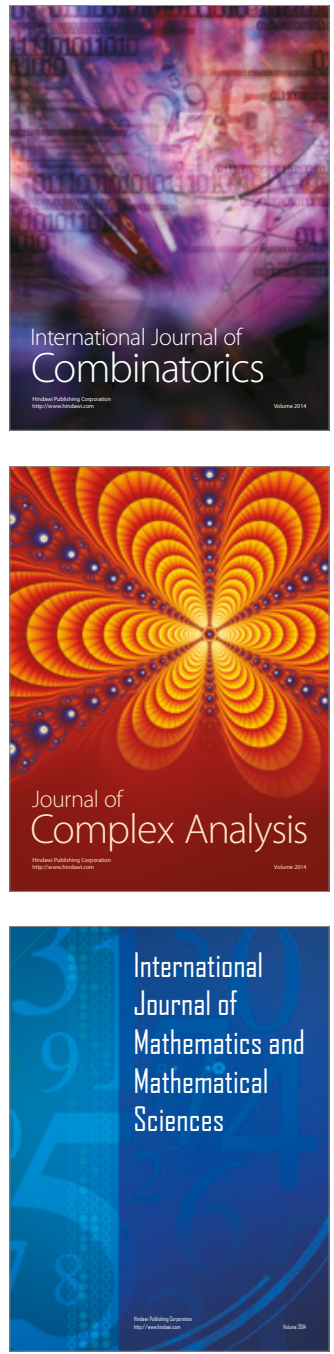
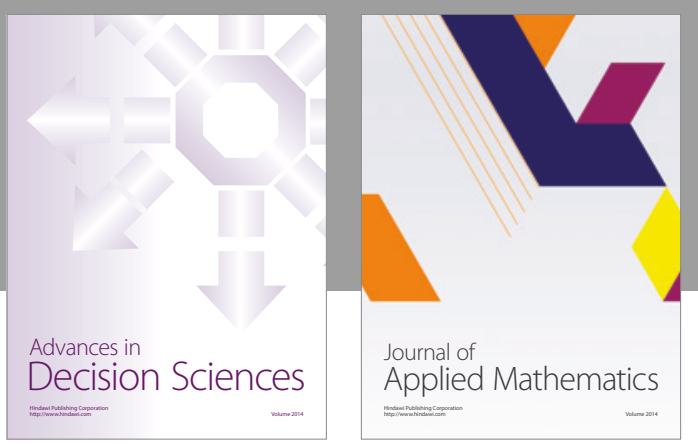

Algebra

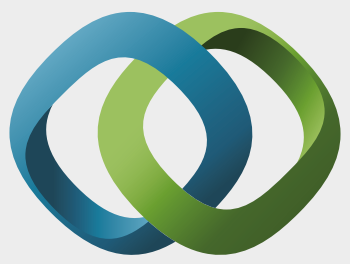

\section{Hindawi}

Submit your manuscripts at

https://www.hindawi.com
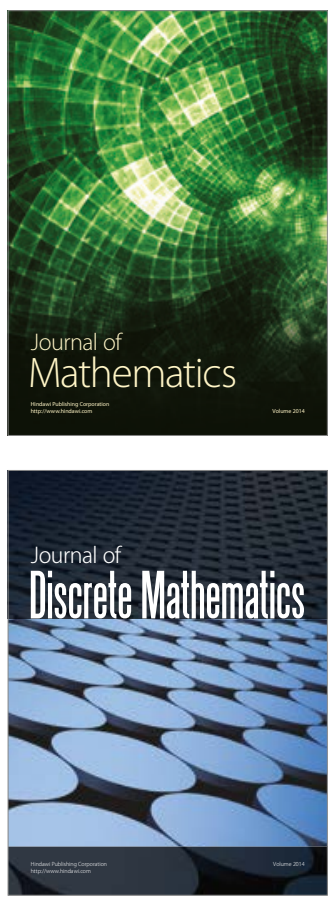

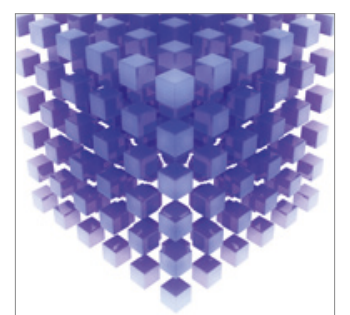

Mathematical Problems in Engineering
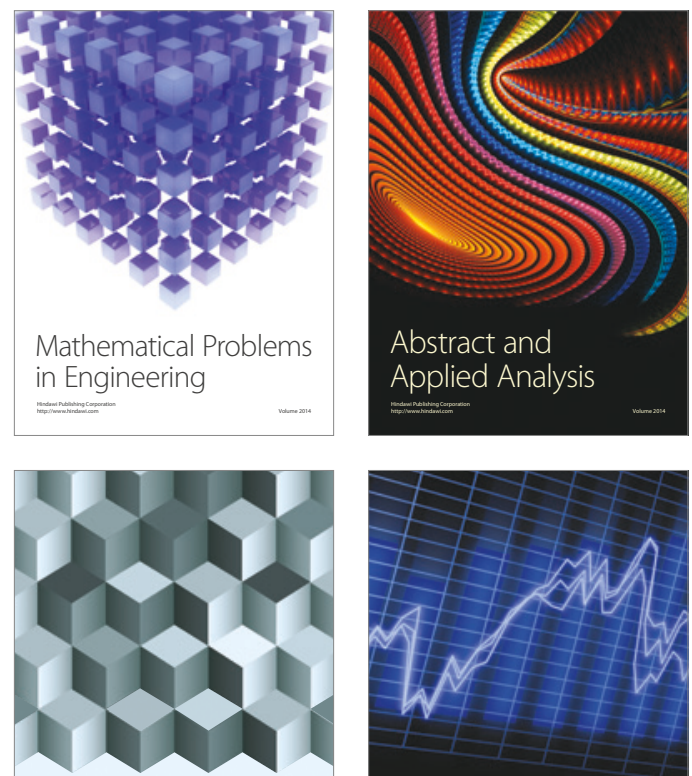

Journal of

Function Spaces

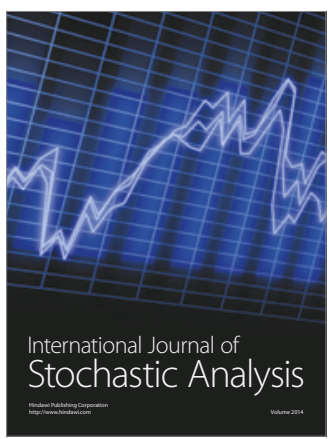

Probability and Statistics
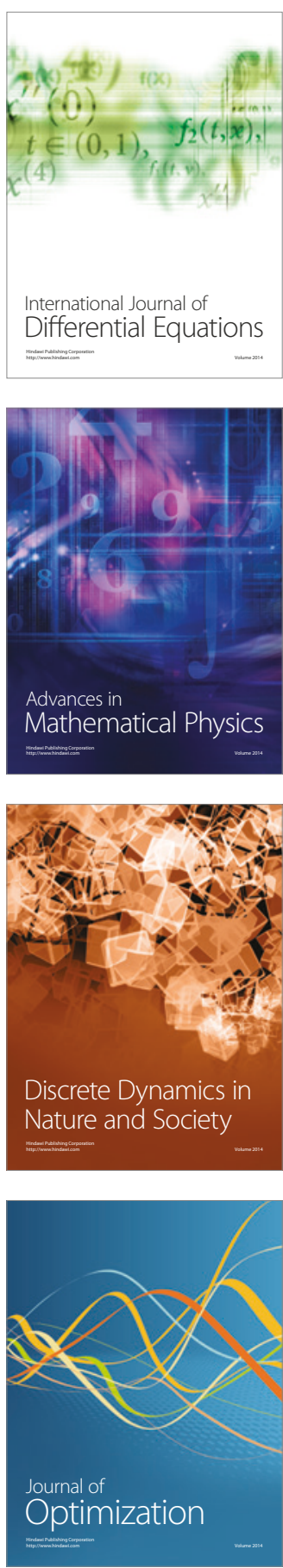